\title{
A high-precision laser method for directly and quickly measuring 21 geometric motion errors of three linear axes of computer numerical control machine tools
}

\author{
Fajia Zheng ${ }^{1} \cdot$ Qibo Feng ${ }^{1} \cdot$ Bin Zhang ${ }^{1}$. Jiakun $\mathrm{Li}^{1} \cdot$ Yuqiong Zhao ${ }^{1}$
}

Received: 24 September 2019 / Accepted: 2 July 2020 / Published online: 14 July 2020

(C) The Author(s) 2020

\begin{abstract}
Error compensation has become an important means of improving the manufacturing and processing accuracy of computer numerical control (CNC) machine tools. Quick and precise measurement of the various geometric motion errors (GMEs) of CNC machine tools is crucial. We propose a novel laser method for the efficient and direct high-precision measurement of the 21 GMEs of a three-axis CNC machine tool, or three linear axes of a five-axis tool. A corresponding system was developed, comprising a fiber-coupled laser unit, sensor head, target unit, and beam-steering unit. The beam-steering unit was designed to perform high-accuracy $90^{\circ}$ rotation of the measuring beam, and the target unit was designed to be sensitive to 18 GMEs of the three linear axes. Stability, repeatability, and comparison experiments were conducted to verify the performance of the proposed system. The results showed that the stability of the position error measurement is $\pm 6.3 \mathrm{~nm}$. For straightness error measurement, the stability, repeatability error, and residual are within \pm $60.3 \mathrm{~nm}, \pm 0.5 \mu \mathrm{m}$, and $\pm 0.7 \mu \mathrm{m}$, respectively. These are within \pm 0.12 arcsec, $\pm 0.5 \operatorname{arcsec}$, and $\pm 0.5 \operatorname{arcsec}$ for the pitch and yaw measurements, and within \pm 0.37 arcsec, \pm 1.5 arcsec, and \pm 1.0 arcsec for the roll measurements, respectively. For squareness error measurement, the repeatability error and residual are within $\pm 0.6 \operatorname{arcsec}$ and $\pm 1.6 \operatorname{arcsec}$, respectively. Compared with a laser interferometer, the proposed system can measure the 21 GMEs of a three-axis machine tool with one-step installation. Without accuracy loss, the measurement efficiency is approximately 45 times higher than that of a laser interferometer, thus providing a new quick and accurate measurement method of GMEs and error compensation of CNC machine tools.
\end{abstract}

Keywords Twenty-one geometric motion errors $\cdot$ Laser measurement $\cdot$ Linear axis $\cdot \mathrm{CNC}$ machine tools

\section{Introduction}

Improving the accuracy of computer numerical control (CNC) machine tools has been a long-standing issue in the field of mechanical manufacturing and precision engineering. One of the effective methods of improving the accuracy is error compensation [1, 2]. Jung et al. reduced the geometric motion errors (GMEs) by $90 \%, 78 \%$, and $92 \%$ by compensating for the X-, Y-, and Z-axes, respectively [3]. However, two key challenges exist in the error compensation of $\mathrm{CNC}$ machine tools: measuring all the GMEs accurately and establishing an accurate error compensation model $[4,5]$.

Qibo Feng

qbfeng@bjtu.edu.cn

1 Key Laboratory of Luminescence and Optical Information, Ministry of Education, Beijing Jiaotong University, Beijing 100044, China
For a three-axis CNC machine tool, $21 \mathrm{GMEs}$ need to be measured [6]. A five-axis CNC machine tool has 42 GMEs [7, 8], 21 of which come from its three linear axes. Figure 1 and Table 1 present a $\mathrm{CNC}$ machine tool with three linear axes and 21 GMEs, respectively. As the error compensation models for the three linear axes of machine tools have been well established $[6,9,10]$, it is vital to be able to quickly and accurately measure all the 21 GMEs in the error compensation of $\mathrm{CNC}$ machine tools.

To measure the GMEs of linear axes of CNC machine tools, researchers have carried out long-term extensive studies using many different and available measurement methods. These methods can be classified into two categories: direct and indirect [11]. Indirect measurement methods require multi-axis motion of the machine under testing, including an artifact test [12, 13], an R test [14, 15], a ball bar test [16-18], and a tracking interferometer [19-21]. Conventionally, these methods have advantages such as high measurement efficiency and simple measurement configuration. However, they 


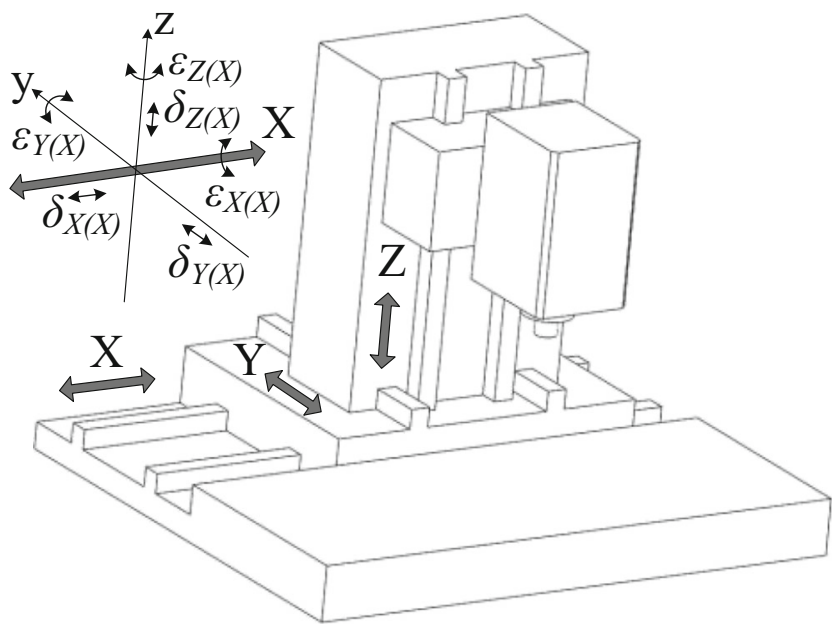

Fig. 1 Three-axis CNC machine tool with six GMEs on the $\mathrm{X}$-axis

require a complex error-decoupling model to identify each individual error, making it difficult to measure errors in real time and perform online error compensation [11,22]. Therefore, direct measurement methods have attracted more attention, as they have high measurement accuracy and can perform online error compensation. A typical direct measurement method is laser interferometry. However, efficiency is a critical issue for interferometry. It normally takes several hours or even days to measure all the errors successively for the three linear axes using a laser interferometer [7, 23, 24]. During the long measurement time, the measurement environment may change significantly. It is therefore difficult to ensure the stability and repeatability of the measurement results, and the overall accuracy is reduced. To improve the measurement efficiency, methods for simultaneously measuring six GMEs of a single linear axis of machine tools have been widely studied. Ni et al. proposed a method based on the combination of laser interferometry and laser collimation for simultaneously measuring multiple GMEs $[25,26]$. Similar methods were introduced by Liu et al. [27, 28]. Wang et al. presented a method based on laser collimation for a miniaturized machine tool [29]. Gao et al. proposed a measurement system using a surface encoder [30, 31]. Fan et al. built a 5D/

Table 1 The 21 GMEs of a three-axis CNC machine tool

\begin{tabular}{llll}
\hline Error & X-axis & Y-axis & Z-axis \\
\hline Position error & $\delta_{X(X)}$ & $\delta_{Y(Y)}$ & $\delta_{Z(Z)}$ \\
Horizontal straightness & $\delta_{Y(X)}$ & $\delta_{X(Y)}$ & $\delta_{Y(Z)}$ \\
Vertical straightness & $\delta_{Z(X)}$ & $\delta_{Z(Y)}$ & $\delta_{X(Z)}$ \\
Yaw & $\varepsilon_{Z(X)}$ & $\varepsilon_{Z(Y)}$ & $\varepsilon_{X(Z)}$ \\
Pitch & $\varepsilon_{Y(X)}$ & $\varepsilon_{X(Y)}$ & $\varepsilon_{Y(Z)}$ \\
Roll & $\varepsilon_{X(X)}$ & $\varepsilon_{Y(Y)}$ & $\varepsilon_{Z(Z)}$ \\
Squareness error & $S_{X Y}, S_{Z X}, S_{Y Z}$ & & \\
\hline
\end{tabular}

6D measurement system consisting of several sets of laser Doppler measuring instruments and semiconductor lasers $[32,33]$. A method using a diffraction grating was proposed by Kim et al. [34] and Liu et al. [35]. A diffraction laser encoder was used by Lee et al. [36]. A commercial 5D/6D measurement instrument was developed by API [37]. Renishaw recently introduced a laser 6D simultaneous measurement system [38]. Additionally, multi-axis laser interferometer systems were introduced by JEANer Meßtechnik $\mathrm{GmbH}$, and Zygo to realize multiple-GME simultaneous measurement of two or three linear axes [39, 40]. However, these direct measurement methods and instruments cannot measure the 21 total GMEs of three-axis CNC machine tools or the three linear axes of five-axis tools.

We proposed a series of direct methods for simultaneously measuring six GMEs of a single linear axis and developed the corresponding systems [41-44]. These methods have been proven to be effective and accurate. Based on these, in this paper, a special beam-steering unit and target unit were invented and developed, and the 21 GMEs of the three linear axes of CNC machine tools can be directly obtained with only one-step installation and three-step automatic measurements for the first time. The beam-steering unit was controlled to perform $90^{\circ}$ rotation of the measuring beam with high accuracy, and the target unit can be sensitive to 18 GMEs of the three linear axes. The corresponding system was then developed. The system is simple and convenient to operate. Compared with a laser interferometer, which is a singleparameter measurement instrument, the proposed system can measure the 21 GMEs of a three-axis CNC machine tool with only one-step installation. Without accuracy loss, the measurement efficiency is approximately 45 times higher than that of a laser interferometer, thus providing a new method for quick and accurate measurements of the GMEs and error compensation of CNC machine tools. The rest of this paper is organized as follows: the proposed method and system are described in Sect. 2, the results of experiments performed to verify the performance of the proposed system are presented in Sect. 3, and the conclusions are given in Sect. 4.

\section{Measurement system configuration and measurement principles}

\subsection{System configuration}

Figure 2 schematically shows the system configuration for measuring the 21 GMEs. The system consists of a fibercoupled laser unit, a sensor head, a target unit, and a beamsteering unit. The target unit, which was invented to be sensitive to 18 GMEs of the three linear axes, mainly comprises three mutually perpendicular six-error sensing components that are each sensitive to six GMEs of the X-, Y-, and Z-axes, 


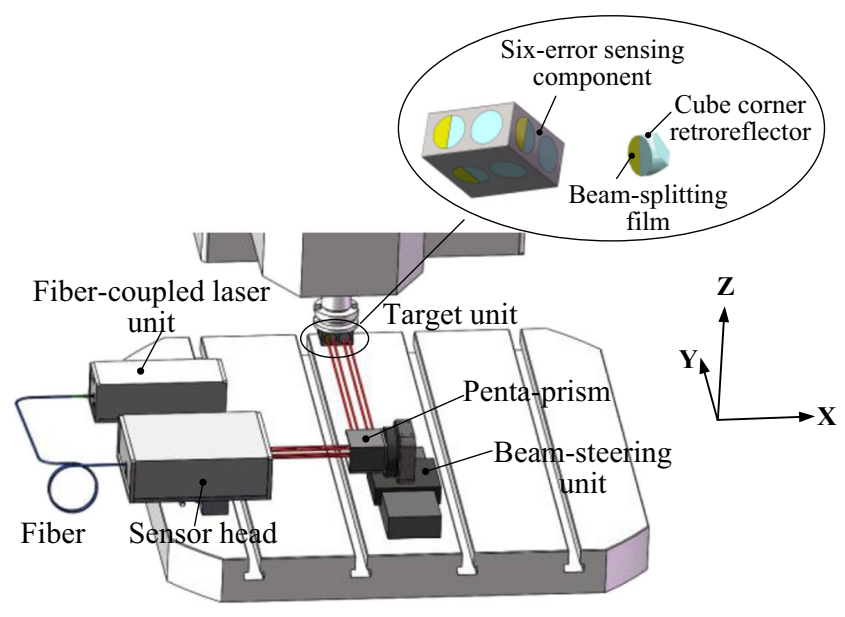

Fig. 2 System configuration for measuring 21 GEMs

respectively. Every six-error sensing component includes two cube corner retroreflectors, one of which is coated with a beam-splitting film on the half-aperture. The main component of the beam-steering unit is a penta-prism. By bringing the penta-prism into the optical path and rotating it by $90^{\circ}$, we can accurately make the laser beam parallel to the Y- and Zaxes, respectively.

Figure 3 shows the optical configuration for simultaneously measuring six GMEs of a single linear axis of a machine tool. The two cube corner retroreflectors (RR2 and RR3) and the beam-splitting film (BS4) comprise a six-error sensing component. The two orthogonal linearly polarized lights, which are produced by a dual-frequency laser source, are coupled into a polarization-maintaining fiber (PMF) through a gradient-index lens. The use of the PMF can provide two benefits. The first is to eliminate the influence of the heat generated by the laser source on the measurement accuracy, and the second is to reduce the volume of the sensor head.
The two orthogonal linearly polarized lights from the PMF are transmitted to the sensor head and collimated by a collimator, and then split into two parts by a beam splitter (BS1). The reflected beam arrives at the detector (D1) through the polarizer (P1) and is used as the reference signal for the position error measurement. The transmitted beam is split into two parts by a polarizing beam splitter (PBS1). The transmitted $\mathrm{p}$ polarized beam from PBS1 is reflected twice by the pentaprism in the beam-steering unit and is controlled to be parallel with the $\mathrm{Y}$-axis direction. It is then reflected by the cube corner retroreflector (RR2) in the six-error sensing component and used for measuring the position error and straightness. The beam transmitted through the beam splitter (BS3) is the measurement beam for the position error measurement. The beam, which is reflected by BS3 and M1, is received by the quadrant detector (QD1) and used to measure the straightness errors.

The s-polarized beam reflected by PBS1 is split into two parts by the beam splitter (BS2). The beam that transmits through BS2 is reflected by the cube corner retroreflector (RR1) and is used as the reference beam for measuring the position error. The measurement and reference beams pass through the polarizer (P2) and interfere; the interference signal is received by the detector (D2). The position error can be obtained by processing the signals of $\mathrm{D} 1$ and $\mathrm{D} 2$. The beam reflected by BS2 is used to measure the straightness, pitch, and yaw. The beam reflected by the beam-splitting film (BS4) in the six-error sensing component is focused on the positionsensitive detector (PSD) by the lens and used to measure the pitch and yaw. The beam that transmits through BS4 is reflected by the cube corner retroreflector (RR3) and detected by the quadrant detector (QD2) to measure the straightness errors. The roll can be obtained from the results of QD1 and QD2.
Fig. 3 Optical configuration for simultaneously measuring six GMEs of a single linear axis

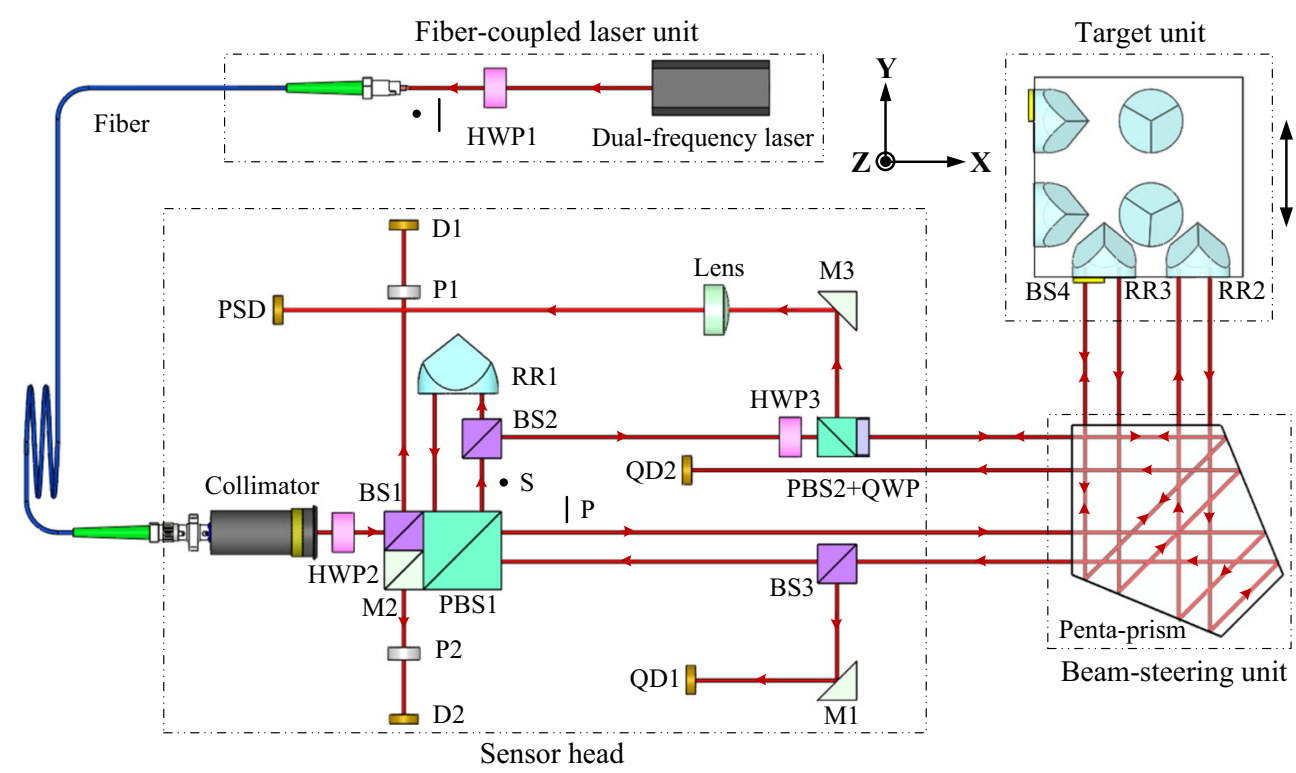


As shown in Fig. 3, the two half-wave plates (HWP1 and HWP2) are used to avoid polarization mixing in the PMF and PBS1, respectively. The half-wave plate (HWP3), the polarizing beam splitter (PBS2), and the quarter-wave plate (QWP) are used to change the polarization direction of the light reflected from BS4 to ensure that the beam is totally reflected by PBS2.

Theoretically, the six GMEs of the Y-axis can be obtained as follows [43]:

$$
\begin{aligned}
\delta_{Y(Y)} & =\frac{\lambda \Delta \phi}{4 n \pi}-L^{\prime} \\
\delta_{X(Y)} & =\frac{\Delta Y_{Q D I}}{2} \\
\delta_{Z(Y)} & =\frac{\Delta Z_{Q D I}}{2} \\
\varepsilon_{X(Y)} & =\frac{\Delta Z_{P S D}}{2 f} \\
\varepsilon_{Z(Y)} & =\frac{\Delta Y_{P S D}}{2 f} \\
\varepsilon_{Y(Y)} & =\frac{\Delta Z_{Q D 2}-\Delta Z_{Q D I}}{2 h}
\end{aligned}
$$

Here, $\lambda$ is the wavelength of the laser beam; $\Delta \phi$ is the phase difference between the reference and measuring beams; $L^{\prime}$ is the nominal displacement of the driven linear guide; $\Delta Y_{Q D 1}$ and $\Delta Z_{Q D 1}$ are the reading changes of QD1 in the $\mathrm{Y}$ - and Z-axis directions, respectively; $\Delta Z_{Q D 2}$ is the reading change of QD2 in the Z-axis direction; $\Delta Y_{P S D}$ and $\Delta Z_{P S D}$ are the reading changes of PSD in the $\mathrm{Y}$ - and $\mathrm{Z}$-axis directions, respectively; $f$ is the focal length of the lens; and $h$ is the distance between the two retroreflectors in the six-error sensing component.

\subsection{Method for measuring 21 GMEs of three linear axes}

Based on the method for simultaneously measuring six GMEs of a single linear axis of a machine tool, as shown in Fig. 3, the 21 GMEs of three linear axes of a machine tool can be measured by one-step installation and three-step measurements [45]. As shown in Fig. 4, the sensor head and the beamsteering unit are both fixed on the clamping workpiece part of the CNC machine tool, and the target unit is fixed on the clamping tool part. The $X-, Y$-, and $Z$-axes of the CNC machine tool are adjusted to the initial measuring position to meet the requirements of ISO230-1 or other relevant measurement standards. First, the penta-prism in the beam-steering unit is moved out of the optical path, as shown in Fig. 4a, and the measuring beam can be adjusted to be parallel to the $\mathrm{X}$-axis. The measurement can be conducted point-by-point, and the six GMEs of all the measuring points on the $\mathrm{X}$-axis are obtained. The penta-prism is then automatically moved into the optical path using an electro-control translation stage, as shown in Fig. 4b. A measuring beam parallel to the $\mathrm{Y}$-axis is obtained, and the six GMEs of all the measuring points on the Y-axis can be obtained in a similar way. The electrocontrol turntable automatically rotates the penta-prism by $90^{\circ}$, as shown in Fig. $4 \mathrm{c}$; thus, the measuring beam is parallel to the Z-axis, and the six GMEs of all the measuring points on the $\mathrm{Z}$-axis are determined. Based on the straightness error of every measuring point on the $\mathrm{X}$-, $\mathrm{Y}$-, and $\mathrm{Z}$-axes, three fitting axes are obtained. The angular error of the penta-prism can be measured in advance, and the squareness errors of each pair on the three axes can be calculated based on these fitting lines and the angular error of the penta-prism. In total, 21 GMEs of the three linear axes of machine tools can be measured.

There are four configurations for the three linear axes of machine tools: TXYZ, XTYZ, XYTZ, and XYZT. The machine tool shown in Fig. 4 is a TXYZ type. In this type of machine tool, there is only one typical measurement mode for measuring the three linear axes, which is that the target unit moves along the linear axis under testing, and the sensor head and beam-steering unit are kept stationary. To measure the 21 GMEs of the other three configurations, there are two other measurement modes. In the first, the sensor head moves along the linear axis under testing, and the target unit and beamsteering unit are kept stationary. In the second, the sensor head and beam-steering unit move along the linear axis under testing, and the target unit is kept stationary. The proposed method can be applied to the measurement for all four configurations by establishing different measurement models. Because of the limited length of this paper, no detailed description is given.

As described above, the proposed measurement method is capable of directly measuring the 21 total GMEs of three linear axes of $\mathrm{CNC}$ machine tools with only one-step installation. Compared with the existing direct measurement methods and instruments, especially with a typical laser interferometer, the proposed measurement method has three obvious advantages. The first is that the proposed measurement method can measure all 21 GMEs of the three linear axes, whereas a laser interferometer can only measure 18 as it cannot measure the roll. The second is that the measuring efficiency of the proposed measurement method is very high. The 21 GMEs of the three linear axes can be quickly measured by one-step installation and three-step automatic measurements. For a laser interferometer, which is a single-parameter measurement instrument, different optical components need to be installed for each error measurement, and the measurement system needs to be re-adjusted, making the measurement process very timeconsuming. The third is that the overall measurement accuracy of the proposed measurement method is high. It can obtain the 21 GMEs of the three linear axes within $10 \mathrm{~min}$, and any change in the measurement environment has little effect on the measurement accuracy, ensuring a higher overall 
Fig. 4 Schematic diagram for measuring 21 GMEs of TXYZ. a six GMEs of $\mathrm{X}$-axis measurement, $\mathbf{b}$ six GMEs of Yaxis measurement, and $\mathbf{c}$ six GMEs of $Z$-axis measurement

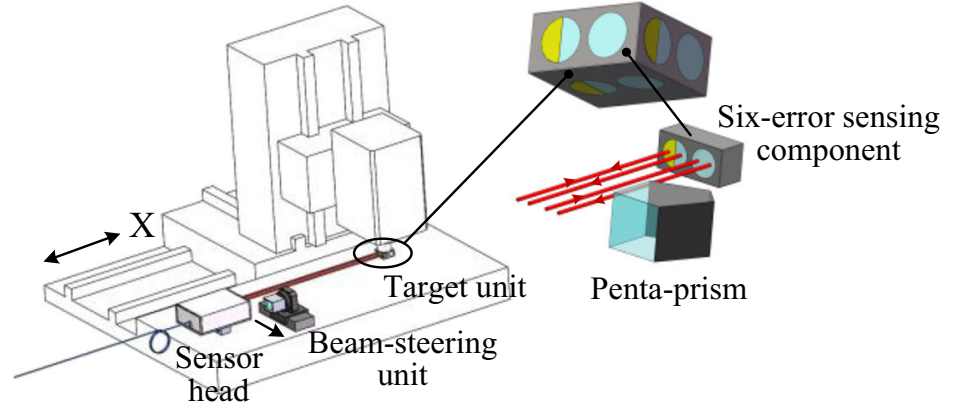

(a)

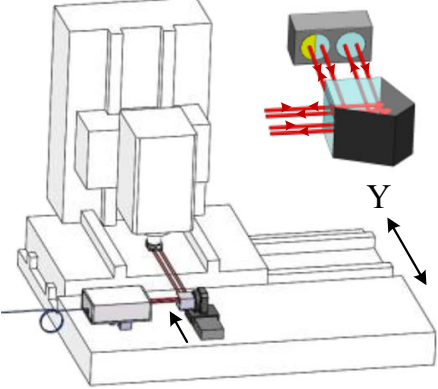

(b)

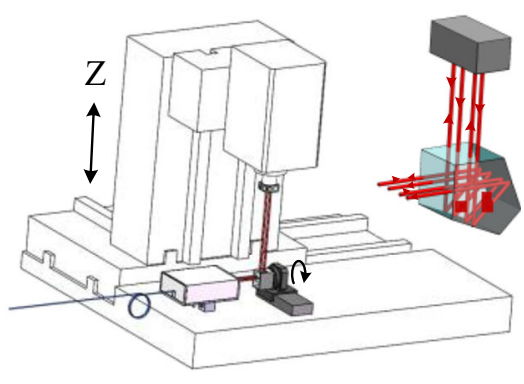

(c) measurement accuracy. For a laser interferometer, although the measurement accuracy is very high, the measurement period is very long, and thus changes in the measurement environment will reduce the overall measurement accuracy. In addition, the measurement error introduced by the multiple installation steps and adjustments will also reduce the overall measurement accuracy of the laser interferometer.

\section{Experimental results and analysis}

A corresponding measurement system was developed. The detectors consist of two QDs (S4349, Hamamatsu) and a PSD (DL16-7-PCBA3, First Sensor). A commercial heterodyne interferometer (LH2000, Leice Technology, accuracy $0.4 \mathrm{ppm}$ ), which can measure at a speed up to $2 \mathrm{~m} / \mathrm{s}$, was integrated into the system, and the two orthogonal linearly polarized lasers generated by the LH2000 were coupled into a PMF (PMJ-633, OZ Optics) to provide the laser source of the measurement system. Thus, the influence of the heat generated by the laser source on the measurement accuracy can be eliminated. In addition, the volume of the sensor head can be reduced.

A target unit, which can be sensitive to 18 GMEs of the three linear axes, was invented and designed. As shown in Fig. 5 , to improve the manufacturing accuracy of the target unit, six cube corner retroreflectors (PS975, Thorlabs, accuracy 3 arcsec), three of which were coated with a beam-splitting film on the half-aperture, were bonded to the three surfaces of a metal base (aluminum). The base was machined with high precision and the squareness error of the two adjacent bonding surfaces is less than 10 arcsec. The developed target unit is small, lightweight, and has no electronic connections, which are convenient for in situ measurements.

A translation stage (ANT130L-110, Aerotech, accuracy $300 \mathrm{~nm}$ ), a turntable (ANT95R-360, Aerotech, accuracy 3 arcsec), and a penta-prism formed the beam-steering unit, wherein the translation stage and turntable were employed to drive the penta-prism in high-precision linear and rotary motions, respectively. As it is the key component in the beam steering unit, the machining accuracy of the penta-prism is critical to the measurement accuracy of the proposed measurement system, especially for the squareness error measurement. Thus, the penta-prism should have high precision. In addition, the maximum distance between the four measuring beams shown in Fig. 3 is designed to be $42 \mathrm{~mm}$ and the diameter

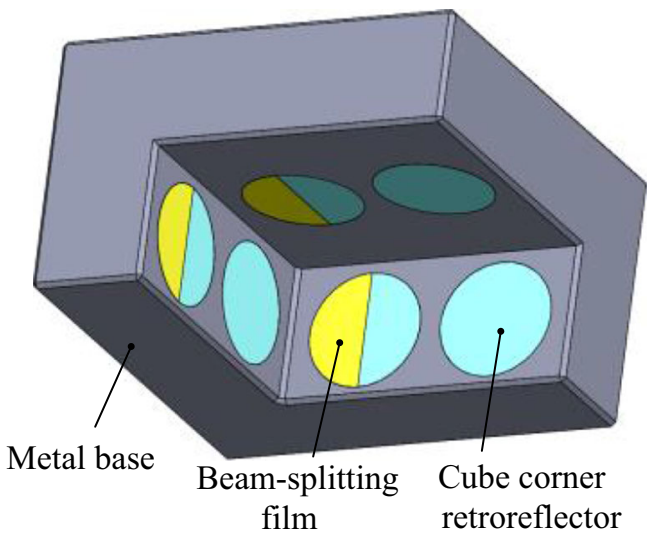

Fig. 5 Target unit 
of the measuring beam is $5 \mathrm{~mm}$. Thus, the clear aperture of the penta-prism should be greater than $47 \mathrm{~mm}$. In this case, the conventional penta-prism is generally heavy and low-accuracy. Thus, to solve these problems, a hollow penta-prism, utilized to rotate the measuring beam by $90^{\circ}$, was designed. As shown in Fig. 6, two reflecting mirrors, coated with reflecting film on the reflecting surface, were bonded to an optical base (N-BK7), and the angle between the two mirrors was precisely controlled at $45^{\circ}$. The developed hollow penta-prism has a high precision of 10 arcsec with a clear aperture of $55 \mathrm{~mm}$, and a light weight of $1.5 \mathrm{~kg}$, which allows it to be fixed on the high-precision turntable ANT95R-360 with a low load.

A series of experiments were carried out to verify the performance of the proposed system.

\subsection{Stability experiments}

In the developed system, the QD and PSD have good linearity in the ranges of \pm 200 and $\pm 600 \mu \mathrm{m}$, respectively. Therefore, the measurement range of the straightness errors is $\pm 100 \mu \mathrm{m}$. As the focal length of the lens is $300 \mathrm{~mm}$, the measurement range of the pitch and yaw is \pm 200 arcsec according to Eq. (1). The distance between the two retroreflectors in the sixerror sensing component is $30 \mathrm{~mm}$; thus, the measurement range of the roll is approximately \pm 680 arcsec according to Eq. (1). For the position error measurement, the measurement range mainly depends on the collimation characteristic of the laser beam and is not less than $2.5 \mathrm{~m}$.

The stability experiment was conducted in 30 min under laboratory conditions, and the variation range of the air temperature was about $\pm 0.2{ }^{\circ} \mathrm{C}$. The target unit was $200 \mathrm{~mm}$ away from the sensor head. The position error was obtained from the interferometer. The detector reading was received via Bluetooth, and the other five GMEs could then be obtained according to Eq. (1). Figure 7 shows the stability results. The

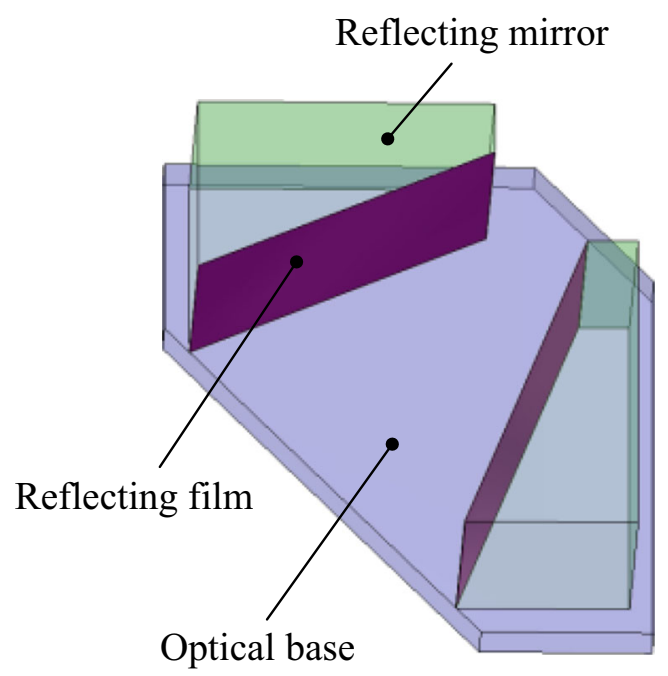

Fig. 6 Hollow penta-prism standard deviations (SDs) of the position error, horizontal straightness, and vertical straightness were 6.3, 13.2, and $60.3 \mathrm{~nm}$, respectively, and the SDs of the yaw, pitch, and roll were $0.09,0.12$, and 0.37 arcsec, respectively. These results show that the proposed system has good stability.

\subsection{Repeatability and comparison experiments}

As shown in Fig. 8a, the 21 GMEs of a three-axis CNC machine tool with XYTZ configuration were measured. Owing to the XYTZ configuration of the machine tool, the motion modes of the three linear axes with respect to the spindle were determined. To measure the 21 GMEs of the three linear axes using the proposed measurement system with one-step installation, the sensor head and beam-steering unit were both fixed on the clamping workpiece part of the machine tool, and the target unit was fixed on the clamping tool part. Thus, there are three measurement modes as mentioned in Sect. 2.2. For the measurement of the six GMEs on the X-axis, the sensor head moves along with the $\mathrm{X}$-axis while the target unit is stationary, as shown in Fig. 8b. For the measurement on the Y-axis, as shown in Fig. 8c, the sensor head and beam-steering unit move along with the $\mathrm{Y}$-axis and the target unit is stationary. For the measurement on the Z-axis, as shown in Fig. 8d, the target unit moves along with the Z-axis, and the sensor head and beam-steering unit are stationary. A commercial laser interferometer (XL80, Renishaw, linear resolution $1 \mathrm{~nm}$, angular resolution $0.01 \mathrm{arcsec}$ ) was used for comparison. It is important to note that the roll cannot be measured by the XL80 interferometer, and an electronic level (WLII, Qianshao, accuracy 0.2 arcsec) was used for roll comparison; however, it cannot be used for the Z-axis measurement.

Five repeated measurements were conducted using the proposed system, and the commercial instruments were then used to measure all the errors successively, except for the roll about the $\mathrm{Z}$-axis and the two squareness errors associated with the Y-axis. Figure 9 shows the results of the comparison experiments, in which the value of each measurement position is the average of five measurements. Table 2 summarizes the results of the repeatability and comparison experiments. Additionally, the measurement times required for the proposed system and commercial instruments were recorded. In the following section, a detailed discussion about the measurement time is presented.

The proposed system and commercial instruments exhibit similar repeatability errors except for the roll, as presented in Table 2 . The repeatability error is half the maximum difference between the maximum and minimum of five experiments at each measuring point. Taking the measurement of yaw on the $\mathrm{X}-, \mathrm{Y}-$, and $\mathrm{Z}$-axes as examples, the repeatability errors of the proposed system are $\pm 0.20, \pm 0.30$, and \pm 0.31 arcsec, respectively, and those of the Renishaw interferometer are $\pm 0.22, \pm 0.15$, and \pm 0.10 arcsec, respectively. The 

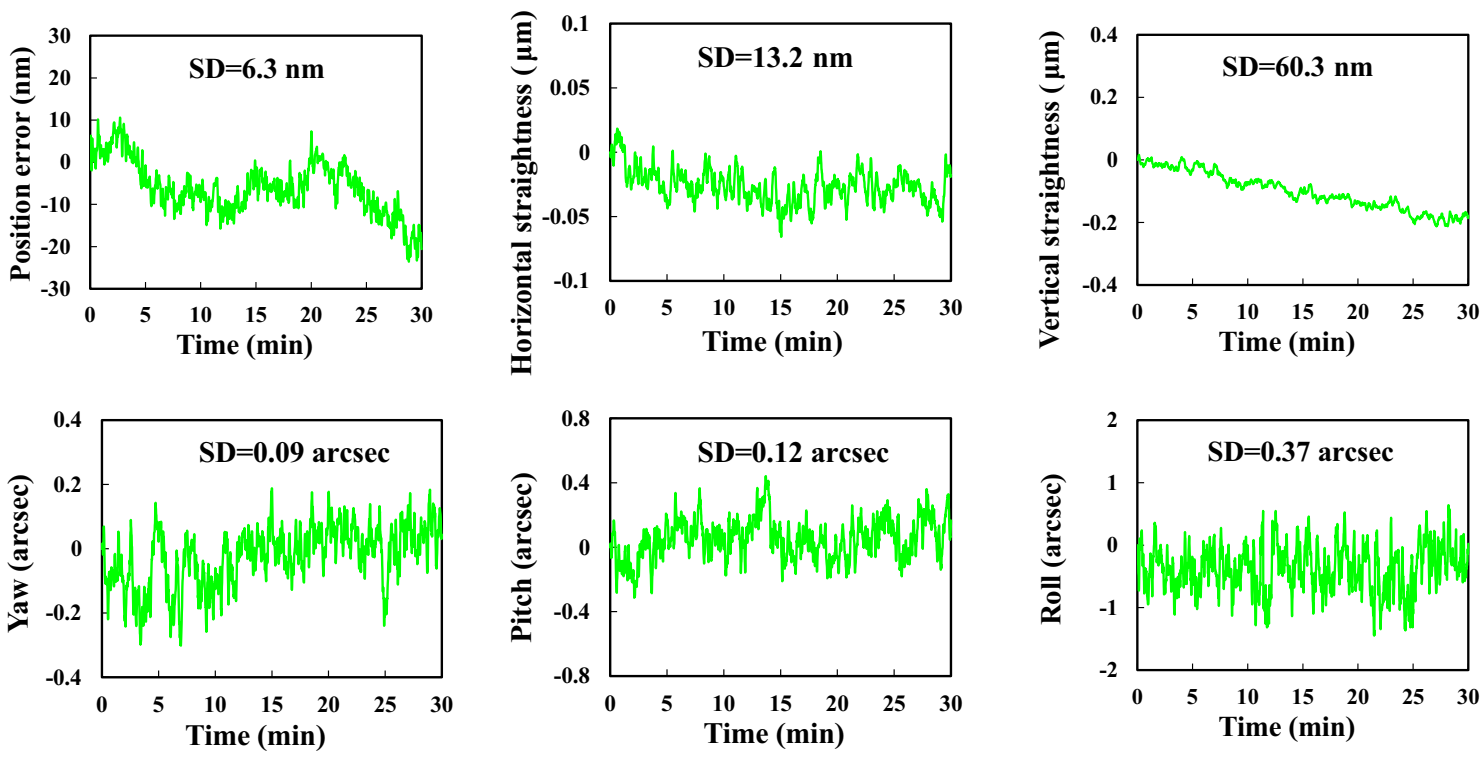

Fig. 7 Results of stability experiments for six GME measurements

primary reason for the higher repeatability error in the roll measurement of the proposed system is the influence of the vertical straightness measurement. The measurement repeatability of the vertical straightness is $\pm 0.1 \mu \mathrm{m}$, and the repeatability of the roll is approximated to \pm 0.67 arcsec in theory, according to Eq. (1).

As shown in Fig. 7 and Table 2, the results of the proposed system are consistent with those obtained using the commercial instruments. For example, for the measurement of vertical straightness on the X-, Y-, and Z-axes, the values of the maximum comparison deviation, which is the maximum deviation between the proposed system and the commercial instrument, are $0.39,0.16$, and $0.65 \mu \mathrm{m}$, respectively. The main reason for the comparison deviation is that the error-sensitive units of the two systems cannot be placed in the same position, thus failing to measure the same point on the linear guide. In addition, the manufacturing and installation errors of the optical elements and the error crosstalk also contribute to the comparison
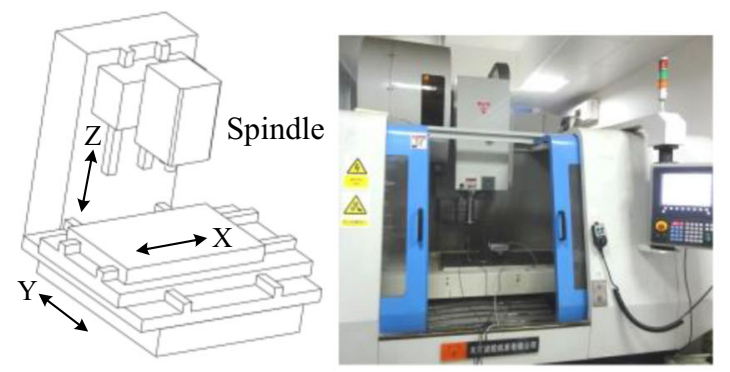

(a)
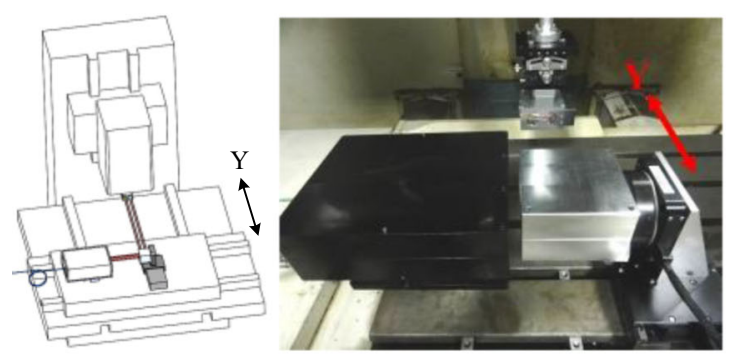

(c)

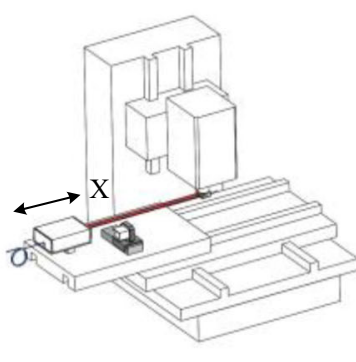

(b)

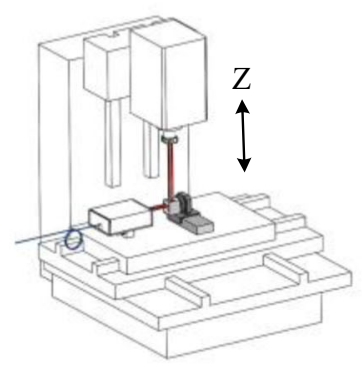

(d)

Fig. 8 Images of the machine tool and experimental setup. a XYTZ machine tool. b Measurement of six GMEs of the X-axis. $\mathbf{c}$ Measurement of six GMEs of the Y-axis. $\mathbf{d}$ Measurement of six GMEs of the Z-axis 


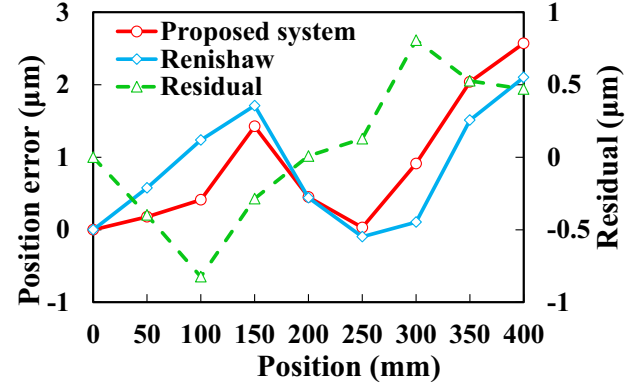

(a)

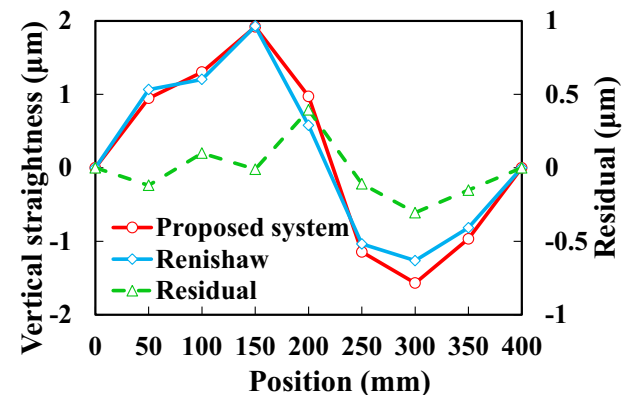

(c)

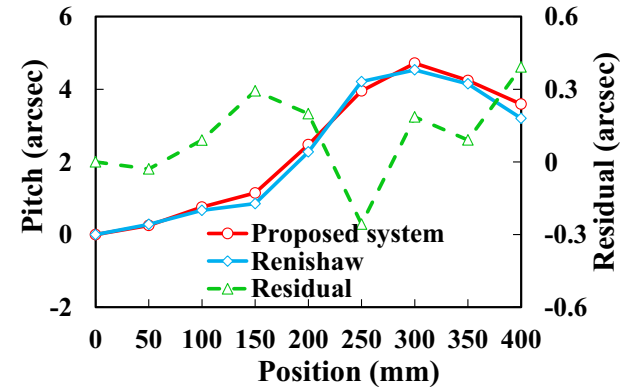

(e)

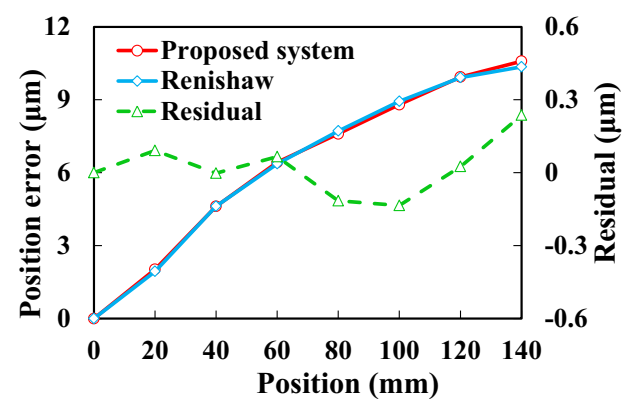

(g)

Fig. 9 Results of comparison experiments. Comparison results of a position error, b horizontal straightness, $\mathbf{c}$ vertical straightness, $\mathbf{d}$ yaw, $\mathbf{e}$ pitch, and $\mathbf{f}$ roll of $\mathrm{X}$-axis; comparison results of $\mathbf{g}$ position error, $\mathbf{h}$ horizontal straightness, $\mathbf{i}$ vertical straightness, $\mathbf{j}$ yaw, $\mathbf{k}$ pitch, and $\mathbf{I}$ roll

deviation; they are very complex and cannot be explained in detail in this paper. We will analyze these errors and establish a compensation model in the future.

Table 2 presents a summary of the performance of the proposed system in measuring the 21 GMEs of the three linear axes of machine tools. For the straightness measurement, the

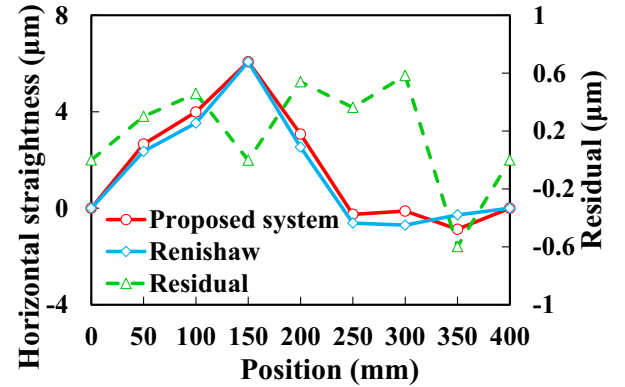

(b)

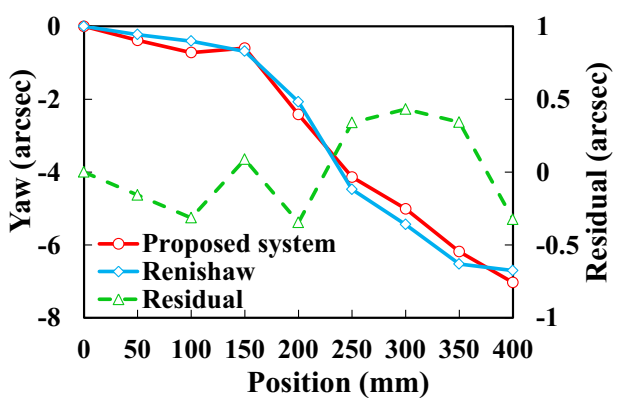

(d)

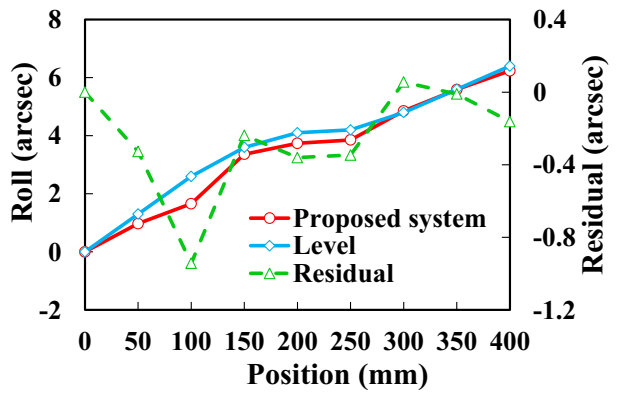

(f)

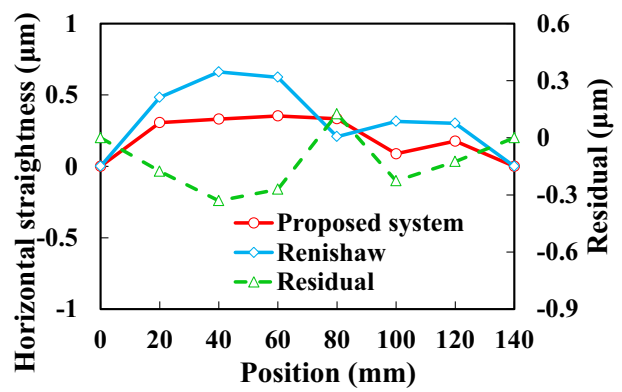

(h)

of Y-axis; comparison results of $\mathbf{m}$ position error, $\mathbf{n}$ horizontal straightness, o vertical straightness, $\mathbf{p}$ yaw, and $\mathbf{q}$ pitch of $\mathrm{Z}$-axis; comparison result of $\mathbf{r}$ squareness error between $\mathrm{X}$ - and Z-axes

repeatability error and residual are within \pm 0.5 and $\pm 0.7 \mu \mathrm{m}$, respectively. For the measurement of yaw and pitch, the repeatability error and residual are within \pm 0.5 and \pm 0.5 arcsec, respectively. For the measurement of roll, the repeatability error and residual are within \pm 1.5 and \pm 1.0 arcsec, respectively. For the measurement of squareness error, the 


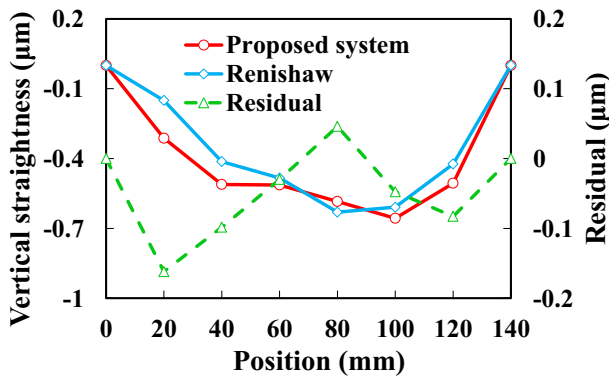

(i)

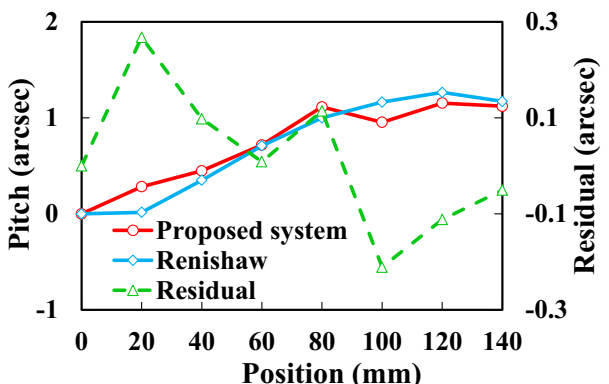

(k)

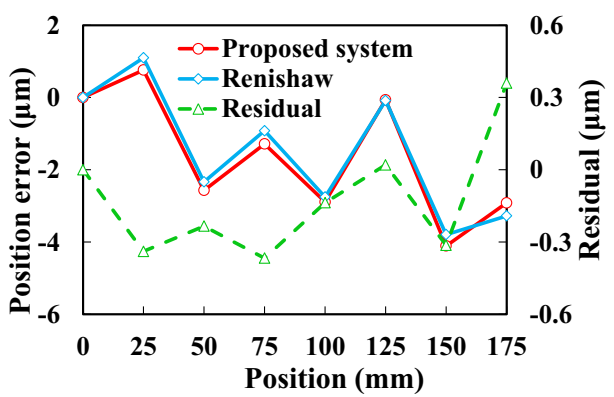

(m)

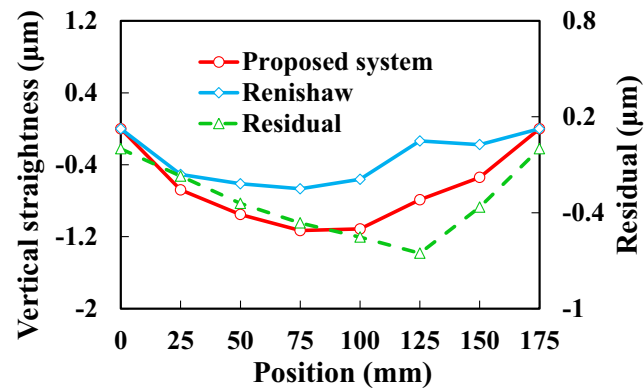

(o)

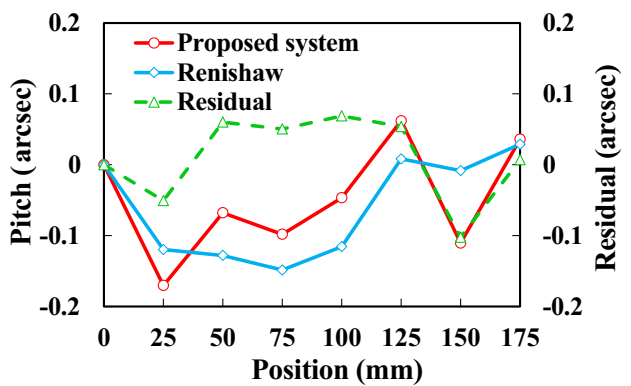

(q)

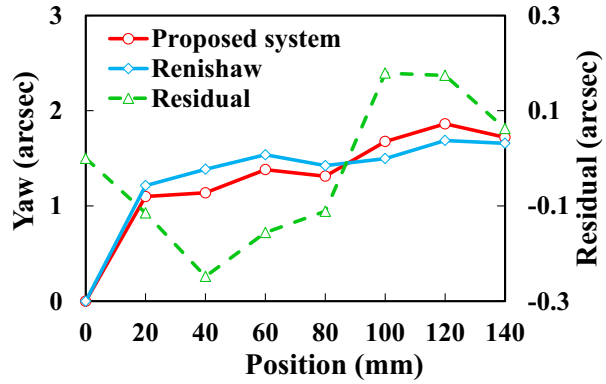

(j)

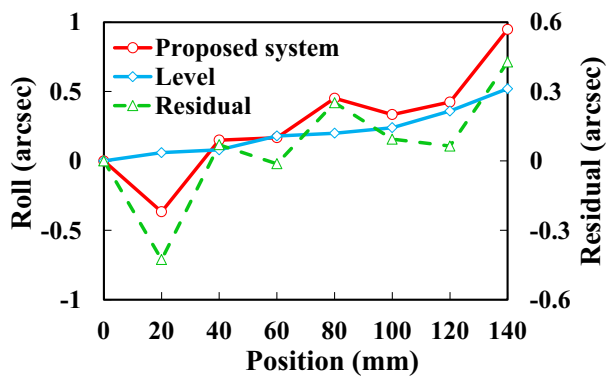

(1)

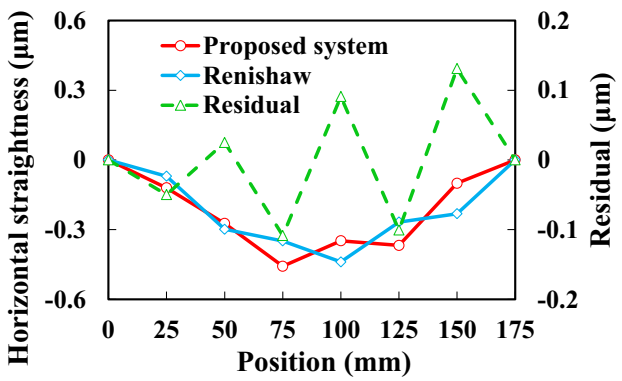

(n)

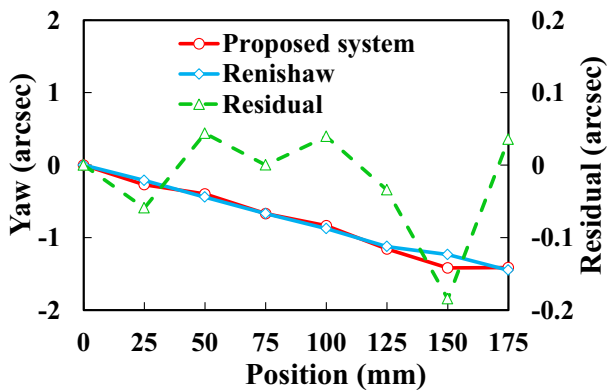

(p)

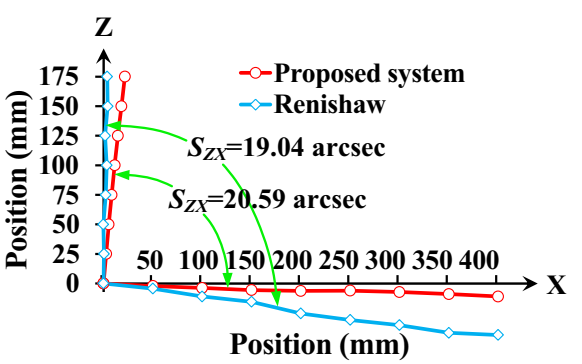

(r)

Fig. 9 continued. 
Table 2 Repeatability error (RE) and maximum comparison deviation (MCD) of the proposed system (PS) and commercial instruments (CIs)

\begin{tabular}{|c|c|c|c|c|c|c|c|c|c|}
\hline \multirow[t]{3}{*}{ Error } & \multicolumn{3}{|l|}{$\mathrm{X}$} & \multicolumn{3}{|l|}{$\mathrm{Y}$} & \multicolumn{3}{|l|}{$\mathrm{Z}$} \\
\hline & \multicolumn{2}{|l|}{$\mathrm{RE}$} & \multirow[t]{2}{*}{ MCD } & \multicolumn{2}{|l|}{$\mathrm{RE}$} & \multirow[t]{2}{*}{ MCD } & \multicolumn{2}{|l|}{$\mathrm{RE}$} & \multirow[t]{2}{*}{ MCD } \\
\hline & PS & CI & & PS & CI & & PS & $\mathrm{CI}$ & \\
\hline Position error $(\mu \mathrm{m})$ & \pm 0.58 & \pm 0.47 & 0.82 & \pm 0.56 & \pm 0.40 & 0.24 & \pm 0.68 & \pm 0.61 & 0.31 \\
\hline $\begin{array}{l}\text { Horizontal straightness } \\
\qquad(\mu \mathrm{m})\end{array}$ & \pm 0.31 & \pm 0.41 & 0.60 & \pm 0.26 & \pm 0.14 & 0.33 & \pm 0.44 & \pm 0.08 & 0.13 \\
\hline $\begin{array}{l}\text { Vertical straightness } \\
\qquad(\mu \mathrm{m})\end{array}$ & \pm 0.25 & \pm 0.13 & 0.39 & \pm 0.30 & \pm 0.08 & 0.16 & \pm 0.37 & \pm 0.04 & 0.65 \\
\hline Yaw $(\operatorname{arcsec})$ & \pm 0.20 & \pm 0.22 & 0.43 & \pm 0.30 & \pm 0.15 & 0.25 & \pm 0.31 & \pm 0.10 & 0.18 \\
\hline Pitch $(\operatorname{arcsec})$ & \pm 0.30 & \pm 0.33 & 0.39 & \pm 0.45 & \pm 0.15 & 0.27 & \pm 0.33 & \pm 0.12 & 0.24 \\
\hline Roll (arcsec) & \pm 1.11 & \pm 0.15 & 0.94 & \pm 1.30 & \pm 0.10 & 0.43 & \pm 1.27 & - & - \\
\hline $\begin{array}{l}\text { Squareness error } S_{Z X} \\
\quad(\operatorname{arcsec})\end{array}$ & \multicolumn{9}{|c|}{ RE of PS: $\pm 0.55 ;$ MCD 1.55} \\
\hline $\begin{array}{l}\text { Squareness error } S_{X Y} \\
\quad(\operatorname{arcsec})\end{array}$ & \multicolumn{9}{|c|}{ RE of PS: \pm 0.27} \\
\hline $\begin{array}{l}\text { Squareness error } S_{Y Z} \\
\quad(\operatorname{arcsec})\end{array}$ & \multicolumn{9}{|c|}{ RE of PS: \pm 0.42} \\
\hline
\end{tabular}

repeatability error and residual are within \pm 0.6 and \pm 1.6 arcsec, respectively. These results demonstrate the high reliability and accuracy of the proposed system.

\subsection{Measurement efficiency comparison}

As shown in Fig. 8, the proposed measurement system can be installed on $\mathrm{CNC}$ machine tools in one step. By adjusting the sensor head, beam-steering unit, and target unit, the beams along the $\mathrm{X}-, \mathrm{Y}-$, and $\mathrm{Z}$-axes are all in the measuring range of the detectors. The installation and adjustment time is approximately $30 \mathrm{~min}$. The measuring distances are 400, 140, and $175 \mathrm{~mm}$ for the X-, Y-, and Z-axes of the CNC machine tool, respectively, and at least eight positions were measured along each linear axis. The feed rate of the machine tool is $600 \mathrm{~mm} / \mathrm{min}$. The measuring time for the three axes is approximately $10 \mathrm{~min}$, which can be reduced to $5 \mathrm{~min}$ by optimizing the measurement procedure in the future. In total, the installation, adjustment and measuring time is approximately $40 \mathrm{~min}$ using the proposed system.

The same machine tool was measured using a Renishaw laser interferometer. In this case, different components need to be installed for each error measurement, and the measurement system needs to be re-adjusted. In addition, as the roll cannot be measured by the interferometer, an electronic level is used. Table 3 lists the total time required for the three-axis measurement. The installation time refers to the time taken to install the different measurement components for each error, the alignment time refers to the time required to adjust the light path to eliminate slanting, and the measuring time is the same as that of the proposed measurement system because the measurement process is controlled by the $\mathrm{CNC}$ machine tool.
The total measurement times for the six GMEs of the Xand $\mathrm{Y}$-axes were 95 and $92 \mathrm{~min}$, respectively. To measure the Z-axis, the installation and adjustment were relatively complicated and difficult, particularly for the straightness measurement, for which an adjustable turning mirror and large retroreflector are required. The time taken to measure the Z-axis was approximately $124 \mathrm{~min}$. The roll about the Z-axis cannot be measured by the electric level. To measure the squareness error between the $\mathrm{X}$ - and $\mathrm{Z}$-axes, up to six optical components are required to be installed and adjusted, and the number of light paths is up to 12 . The installation and adjustment are very complicated and difficult. The time required for measuring the squareness error between the $\mathrm{X}$ - and Z-axes was approximately $54 \mathrm{~min}$, and it took approximately $162 \mathrm{~min}$ to measure the three squareness errors.

Table 3 Installation time (IT), alignment time (AT), and measuring time (MT) of a three-axis machine tool using a Renishaw interferometer and electric level

\begin{tabular}{|c|c|c|c|c|c|c|c|c|c|}
\hline \multirow[t]{2}{*}{ Error } & \multicolumn{3}{|c|}{$\mathrm{X}(\min )$} & \multicolumn{3}{|c|}{$\mathrm{Y}(\min )$} & \multicolumn{3}{|c|}{$\mathrm{Z}(\min )$} \\
\hline & IT & AT & MT & IT & AT & MT & IT & AT & MT \\
\hline Position error & 15 & 10 & 2 & 15 & 10 & 1.5 & 15 & 15 & 1.5 \\
\hline Horizontal straightness & 10 & 8 & 2 & 10 & 8 & 1.5 & 15 & 15 & 1.5 \\
\hline Vertical straightness & 5 & 8 & 2 & 5 & 8 & 1.5 & 10 & 15 & 1.5 \\
\hline Pitch & 10 & 5 & 2 & 10 & 5 & 1.5 & 10 & 8 & 1.5 \\
\hline Yaw & 5 & 5 & 2 & 5 & 5 & 1.5 & 5 & 8 & 1.5 \\
\hline Roll & 1 & 1 & 2 & 1 & 1 & 1.5 & - & - & - \\
\hline Three squareness errors & \multicolumn{9}{|c|}{$162 \mathrm{~min}$} \\
\hline Total & \multicolumn{9}{|c|}{$473 \mathrm{~min}$} \\
\hline
\end{tabular}


The total time required for measuring the 20 GMEs of the same CNC machine tool with three linear axes was approximately 473 min using the laser interferometer and electric level. It is noteworthy that only a one-step installation is required for the proposed system, whereas 20 steps are involved in installing the laser interferometer and electric level. The measurement error due to the multiple installation steps and subsequent adjustments reduces the measurement accuracy. To compare the measurement efficiency of the proposed system with that of the laser interferometer, it is better to exclude the first installation and adjustment time for both the Renishaw and proposed system. Thus, the measurement efficiency of the developed system is approximately 45 times higher than that of the laser interferometer.

In addition, the measurement period of the laser interferometer is long, and the measurement environment changes during this period, which reduces the overall accuracy. In contrast, the proposed high-efficiency measurement method not only saves a significant amount of operation time of the machine tool for measurement but also ensures a higher overall measurement accuracy.

\section{Conclusion}

In this paper, a new method was proposed to efficiently and directly measure the 21 GMEs of a three-axis CNC machine tool, or three linear axes of a five-axis $\mathrm{CNC}$ machine tool, for the first time. A corresponding measurement system was developed, wherein a beam-steering unit was invented and designed to perform $90^{\circ}$ rotation of the measuring beam with high accuracy, and a target unit was invented and developed to be sensitive to 18 GMEs of the three linear axes. The 21 GMEs of the three linear axes can be measured using the proposed system with only one-step installation and threestep automatic measurements. The performance of the proposed system was verified by conducting a series of stability, repeatability, and comparison experiments. Compared with a conventional laser interferometer, which is a single-parameter measurement instrument, the developed measurement system has three obvious advantages. The first is that the developed system can measure the total 21 GMEs of the three linear axes. The second is that the measuring efficiency of the developed system is very high, and is increased by approximately 45 times compared with a conventional laser interferometer. The third is that the overall measurement accuracy of the developed system is high. As it took just $10 \mathrm{~min}$ to complete the measurement of the three linear axes, a change in the measurement environment has little effect on the measurement accuracy. We believe the proposed measurement method provides a new method for quick and accurate measurements of the GMEs and error compensation of $\mathrm{CNC}$ machine tools. In the future, we will focus on system optimization, accuracy improvement, and error compensation of machine tools.

Funding information The authors acknowledge the finical support from the National Natural Science Foundation of the China-Major Program (51527806).

Open Access This article is licensed under a Creative Commons Attribution 4.0 International License, which permits use, sharing, adaptation, distribution and reproduction in any medium or format, as long as you give appropriate credit to the original author(s) and the source, provide a link to the Creative Commons licence, and indicate if changes were made. The images or other third party material in this article are included in the article's Creative Commons licence, unless indicated otherwise in a credit line to the material. If material is not included in the article's Creative Commons licence and your intended use is not permitted by statutory regulation or exceeds the permitted use, you will need to obtain permission directly from the copyright holder. To view a copy of this licence, visit http://creativecommons.org/licenses/by/4.0/.

\section{References}

1. Khan AW, Chen W (2011) A methodology for systematic geometric error compensation in five-axis machine tools. Int J Adv Manuf Technol 53(5-8):615-628

2. Shen H, Fu J, He Y, Yao X (2012) On-line asynchronous compensation methods for static/quasi-static error implemented on CNC machine tools. Int J Mach Tools Manuf 60:14-26

3. Jung JH, Choi JP, Lee SJ (2006) Machining accuracy enhancement by compensating for volumetric errors of a machine tool and onmachine measurement. J Mater Process Technol 174(1-3):56-66

4. Ding S, Huang X, Yu C, Liu X (2016) Novel method for positionindependent geometric error compensation of five-axis orthogonal machine tool based on error motion. Int J Adv Manuf Technol 83: 1069-1078

5. Wang W, Chen Z, Zhu Y, Yang H, Lu K, Shi G, Xiang K, Ju B (2020) Full-scale measurement of CNC machine tools. Int J Adv Manuf Technol 107:2291-2301

6. Okafor AC, Ertekin YM (2000) Derivation of machine tool error models and error compensation procedure for three axes vertical machining center using rigid body kinematics. Int $\mathrm{J}$ Mach Tools Manuf 40(8):1199-1213

7. ISO230-1:2012 Test Code for Machine Tools —Part 1: Geometric accuracy of machines operating under no-load or quasi-static conditions

8. Alessandro V, Gianni C, Antonio S (2015) Axis geometrical errors analysis through a performance test to evaluate kinematic error in a five axis tilting-rotary table machine tool. Precis Eng 39:224-233

9. Hsu YY, Wang SS (2007) A new compensation method for geometry errors of five-axis machine tools. Int $\mathrm{J}$ Mach Tools Manuf 47(2):352-360

10. Xiang S, Altintas Y (2016) Modeling and compensation of volumetric errors for five-axis machine tools. Int J Mach Tools Manuf 101:65-78

11. Schwenke H, Knapp W, Haitjema H, Weckenmann A, Schmitt R, Delbressine F (2008) Geometric error measurement and compensation of machines - an update. CIRP Ann Manuf Technol 57(2): $660-675$

12. Bringmann B, Küng A, Knapp W (2005) A measuring artefact for true 3D machine testing and calibration. CIRP Ann Manuf Technol 54(1):471-474 
13. Trapet E, Aguilar Martín JJ, Yagüe JA, Spaan H, Zelený V (2006) Self-centering probes with parallel kinematics to verify machinetools. Precis Eng 30(2):165-179

14. Hong C, Ibaraki S (2013) Non-contact R-test with laser displacement sensors for error calibration of five-axis machine tools. Precis Eng 37(1):159-171

15. Weikert S (2004) R-test, a new device for accuracy measurements on five axis machine tools. CIRP Ann Manuf Technol 53(1):429_ 432

16. Lu X, Jamalian A (2011) A new method for characterizing axis of rotation radial error motion: part 1. Two-dimensional radial error motion theory. Precis Eng 35(1):73-94

17. Archenti A, Nicolescu M, Casterman G, Hjelm S (2012) A new method for circular testing of machine tools under loaded condition. Procedia CIRP 1:575-580

18. Fan KC, Wang H, Shiou FJ, Ke CW (2004) Design analysis and applications of a 3D laser ball bar for accuracy calibration of multiaxis machines. J Manuf Syst 23(3):194-203

19. Aguado S, Samper D, Santolaria J, Aguilar JJ (2014) Volumetric verification of multiaxis machine tool using laser tracker. Sci World J 2014:959510

20. Wang J, Guo J (2016) Geometric error identification algorithm of numerical control machine tool using a laser tracker. Proc IMechE, Part B: J Engineering Manufacture 230(11):2004-2015

21. Holub M, Vetiska J, Bradac F, Vala M (2017) Application on-thefly measurement of CNC machine tools. MM Sci J 2017(5):20852089

22. Li J, Feng Q, Bao C, Zhang B (2019) Method for simultaneously and directly measuring all six-DOF motion errors of a rotary axis. Chin Opt Lett 17(1):011203

23. Okafor AC, Ertekin YM (2000) Vertical machining center accuracy characterization using laser interferometer: part 1. Linear positional errors. J Mater Process Technol 105(3):394-406

24. Okafor AC, Ertekin YM (2000) Vertical machining center accuracy characterization using laser interferometer: part 2. Angular errors. J Mater Process Technol 105(3):407-420

25. Huang PS, Ni J (1994) On-line error compensation of coordinate measuring machines. Int J Mach Tools Manuf 35(5):725-738

26. Ni J, Huang PS, Wu SM (1992) A multi-degree-of-freedom measuring system for CMM geometric errors. J Eng Ind 114(3):362369

27. Chen YT, Lin WC, Liu CS (2017) Design and experimental verification of novel six-degree-of freedom geometric error measurement system for linear stage. Opt Laser in Eng 92:94-104

28. Liu CS, Lai JJ, Luo YT (2018) Design of a measurement system for six-degree-of-freedom geometric errors of a linear guide of a machine tool. Sensors (Basel) 19(1):5

29. Wang W, Kweon SH, Hwang CS, Kang NC, Kim YS, Yang SH (2008) Development of an optical measuring system for integrated geometric errors of a three-axis miniaturized machine tool. Int $\mathrm{J}$ Adv Manuf Technol 43:701-709
30. Gao W, Dejima S, Shimizu Y, Kiyono S, Yoshikawa H (2003) Precision measurement of two-axis positions and tilt motions using a surface encoder. CIRP Ann Manuf Technol 52(1):435-438

31. Li X, Shimizu Y, Ito T, Cai Y, Ito S, Gao W (2014) Measurement of six-degree-of-freedom planar motions by using a multiprobe surface encoder. Opt Eng 53(12):122405

32. Fan KC, Chen MJ, Huang WM (1998) A six-degree-of-freedom measurement system for the motion accuracy of linear stages. Int J Mach Tools Manuf 38(3):155-164

33. Fan KC, Wang HY, Yang HW, Chen LM (2014) Techniques of multi-degree-of-freedom measurement on the linear motion errors of precision machines. Adv Opt Technol 3(4):375-386

34. Kim JA, Kim KC, Bae EW, Kim S, Kwak YK (2000) Six-degreeof-freedom displacement measurement system using a diffraction grating. Rev Sci Instrum 71(8):3214-3219

35. Liu CH, Jywe WY, Chen CK (2005) Development of a simple system for the simultaneous measurement of pitch, yaw and roll angular errors of a linear stage. Int J Adv Manuf Technol 26:808813

36. Lee C, Lee SK (2013) Multi-degree-of-freedom motion error measurement in an ultraprecision machine using laser encoder-review. J Mech Sci Technol 27(1):141-152

37. Lau KC, Liu YQ (2000) Five-axis/six-axis laser measuring system. US Patent 6049377

38. Angood SM, Kemp C, Chaney RJ, Chapman MAV, Mcmurtry DR (2013) Rotation detection kit. US Patent 8368887

39. JEANer Meßtechnik GmbH (2018) Zweifrequenzlaserinterferometer ZLM 700/ 800/ 900 dual- frequency laser interferometer. https://www.jenaer-mt.com/wp-content/uploads/ 2018/08/Gesamtkatalog_ZLM.pdf. Accessed 26 May 2020

40. Zygo. Displacement measuring interferometers. https://www.zygo. com/?/met/markets/stageposition/zmi/. Accessed 26 May 2020

41. Feng Q, Zhang B, Cui C, Kuang C, Zhai Y, You F (2013) Development of a simple system for simultaneously measuring 6DOF geometric motion errors of a linear guide. Opt Express 21(22):25805-25819

42. Zhao Y, Zhang B, Feng Q (2017) Measurement system and model for simultaneously measuring 6DOF geometric errors. Opt Express 25(18):20993-21007

43. Cui C, Feng Q, Zhang B, Zhao Y (2016) System for simultaneously measuring 6DOF geometric motion errors using a polarization maintaining fiber-coupled dual-frequency laser. Opt Express 24(6):6735-6748

44. Feng Q, Zhang B, Gao Z, Cui C (2018) 6DOF error laser simultaneous measurement system with a single polarization maintaining fiber coupling and transmitting the dual-frequency laser. US Patent 9857161B2

45. Feng Q, Zhang B, Cui C (2018) Laser measurement system and method for measuring 21 GMES. US Patent 9982997B2

Publisher's note Springer Nature remains neutral with regard to jurisdictional claims in published maps and institutional affiliations. 